\title{
Theofilos
}

A Nordic open access journal in Theology, Philosophy and Culture

Published by NLA University College - in partnership with Johannelund School of Theology

Available at www.theofilos.no

\section{How to formulate the fine-tuning argument for the existence of God?}

\author{
Atle Ottesen Søvik \\ Professor in Systematic Theology \\ MF Norwegian School of Theology, Religion, and Society \\ Atle.O.Sovik@mf.no
}

\begin{abstract}
This article argues that the fine-tuning argument for the existence of God should have the following characteristics:

It should be clear and logically valid.

- It should contrast the God hypothesis with a chance hypothesis as opposed to a single naturalistic universe hypothesis.

口 It should use the likelihood approach as opposed to ruling out alternatives.

I It should have a specific concept of God as a hypothesis as opposed to intention or design.

I It should have life-permittingness as opposed to fine-tuning as datum.

Different well-known formulations have different combinations of some of these characteristics, but I defend a new formulation, which has all the characteristics.
\end{abstract}

Keywords: Fine-tuning argument; formulation of the fine-tuning argument; W.L.Craig; Robin Collins; Luke Barnes

\section{Introduction}

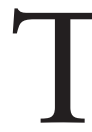

The last couple of decades has seen an increasing interest in debating arguments for and against the existence of God. Numerous debates can be found on YouTube, drawing large crowds. Among the most discussed arguments for the existence of God are cosmological arguments and design arguments. Among design arguments, most attention has been given in recent years to the fine-tuning argument for the existence of God. It is acknowledged by scholars advocating atheism as the best argument for the existence of God. ${ }^{1}$

The fine-tuning argument points to evidence indicating design. It is based on findings from physics showing that many laws of nature, forces, physical parameters and initial conditions of the universe must have very exact values in order for life to be possible. This is called finetuning, but the concept does not imply that anyone actually has fine-tuned anything, it just means that of all the possible universes, the subset that contains life is very small. For example, Roger Penrose has argued that the entropy of the early universe must have been very low, but as far as we know it could have been anything. Penrose calculates it to be finetuned to $1: 10$ raised to $10^{123} .^{2}$

Examples like this show that it is highly unlikely for life to have come 
about by chance, and so it can be used to argue that life is not a result of chance, but instead a result of design. But what is the best way to formulate such an argument for the existence of God? That is the topic for this article.

The fine-tuning argument for the existence of God has so far mainly been about fine-tuning for the existence of life. However, in a forthcoming book, Robin Collins uses fine-tuning for discoverability as an argument for the existence of God. The present article is about finetuning for the existence of life and how this can be used to formulate an argument for the existence of God, so this is what will be meant by the fine-tuning argument from now on.

Probably the two most famous defenders of the fine-tuning argument are Robin Collins and William Lane Craig. ${ }^{3}$ They formulate the argument in quite different ways. Craig offers the following formulation:

P1: Fine-tuning is due to either physical necessity, chance or design.

P2: Fine-tuning is not due to physical necessity or chance.

C: Therefore, it is due to design. ${ }^{4}$

Collins, on the other hand, formulates it this way:

(LPU=The existence of a life-permitting universe; NSU=The naturalistic single universe hypothesis; $\mathrm{T}=\mathrm{The}$ Theistic hypothesis, $\mathrm{P}=$ epistemic probability, k'=some appropriately chosen background information)

1) Given the fine-tuning evidence, LPU is very, very epistemically unlikely under NSU: that is, $\mathrm{P}\left(\right.$ LPUINSU \& $\left.\mathrm{k}^{\prime}\right)<1$, where $\mathrm{k}$ represents some appropriately chosen background information, and $<$ represents much, much less than (thus making P(LPUINSU $\& \mathrm{k}^{\prime}$ ) close to zero).

2) Given the fine-tuning evidence, LPU is not unlikely under theism: that is, $\sim \mathrm{P}\left(\right.$ LPUIT $\left.\& \mathrm{k}^{\prime}\right)<1$. 3) $T$ was advocated prior to the fine-tuning evidence (and has independent motivation).

4) Therefore, by the restricted version of the likelihood principle, LPU strongly supports T over NSU. 5

A far lesser known but very interesting formulation is offered by Norwegian philosopher Einar Duenger Bøhn, who formulates it this way:

( $\mathrm{Pr}=$ Probability, F=Fine-tuning, I=Intention, $\mathrm{C}=$ Chance)

1) $\operatorname{Pr}(\mathrm{F} \mid \mathrm{I})>\operatorname{Pr}(\mathrm{F} \mid \mathrm{C}) \quad \mathrm{L}(\mathrm{I} \mid \mathrm{F})>\mathrm{L}(\mathrm{C} \mid \mathrm{F})$

(Instance of the likelihood principle)

2) $\operatorname{Pr}(\mathrm{F} \mid \mathrm{I})>\operatorname{Pr}(\mathrm{F} \mid \mathrm{C})$

(Premise)

3) $\mathrm{L}(\mathrm{I} \mid \mathrm{F})>\mathrm{L}(\mathrm{ClF})$

$(1,2, \mathrm{MP})^{6}$

In full sentences, the argument says that if the probability of fine-tuning given intention is greater than the probability of finetuning given chance, then the likelihood of an intention given fine-tuning is greater than the likelihood of chance given finetuning. This follows from the so-called likelihood principle, which says that if hypothesis A makes us expect the data we have to a larger degree than hypothesis B, then the data counts as evidence in favour of hypothesis A.7 Since in fact the probability of fine-tuning given intention is more probable that the probability of fine-tuning given chance, it follows logically (by modus ponens) that the likeli- 
hood of an intention given fine-tuning is greater than the likelihood of chance given fine-tuning.

In this article I shall argue that the finetuning argument for the existence of God should have the following characteristics:

A) It should be clear

B) It should be logically valid

C) It should contrast the God hypothesis with a chance hypothesis as opposed to a naturalistic single-universe hypothesis, since that allows us to treat both the universe and the multiverse hypotheses when discussing the second premise.

D) It should use the likelihood approach as opposed to ruling out alternatives, since ruling out alternatives is more difficult.

E) It should have a specific concept of God as a hypothesis as opposed to just intention or design, since it is less clear what intention or design implies.

F) It should have life-permittingness as datum as opposed to fine-tuning, since fine-tuning does not follow from theism, design or intention, but life-permittingness follows from a specific God hypothesis.

Considering the formulations presented so far, William Lane Craig has A, B, C, but not D, E and F. Robin Collins has B, D, E, F, but not $\mathrm{A}$ and C. Einar Duenger Bøhn has A, B, C, D, but not E and $F$. In this article I shall suggest a new formulation which has all characteristics A-F. A and B are taken from Bøhn, C from Craig and Bøhn, D from Bøhn and Collins, and $\mathrm{E}$ and $\mathrm{F}$ from Collins.

The new formulation suggested is like this:

$\mathrm{L}=$ Life-permittingness, $\mathrm{T}=$ Theism, $\mathrm{C}=$ Chance, $\operatorname{Pr}=$ Probability

1) $\operatorname{Pr}(\mathrm{L} \mid \mathrm{T})>\operatorname{Pr}(\mathrm{L} \mid \mathrm{C}) \quad \operatorname{Pr}(\mathrm{T} \mid \mathrm{L})>\operatorname{Pr}(\mathrm{Cl} / \mathrm{L})$ (Instance of the likelihood principle)
2) $\operatorname{Pr}(\mathrm{L} \mid \mathrm{T})>\operatorname{Pr}(\mathrm{L} \mid \mathrm{C})$

(Premise)

3) $\operatorname{Pr}(\mathrm{T} \mid \mathrm{L})>\operatorname{Pr}(\mathrm{ClL})$

$(1,2, \mathrm{MP})$

In full sentences, the argument says that if the probability of life-permittingness given theism is greater than the probability of life-permittingness given a chance hypothesis, then the likelihood of theism given life-permittingness is greater than the likelihood of a chance hypothesis given life-permittingness. Since in fact the probability of life-permittingness given theism is more probable that the probability of life-permittingness given a chance hypothesis, it follows that the likelihood of theism given life-permittingness is greater than the likelihood of a chance hypothesis given life-permittingness.

When I first submitted this article to Theofilos, Luke Barnes had not yet published his article 'A Reasonable Little Question: A Formulation of the FineTuning Argument'. ${ }^{8}$ In that article, he recommends a formulation of the argument, which is quite similar to the one I suggest. His formulation is as follows:

[1] For two theories T1 and T2, in the context of background information $\mathrm{B}$, if it is true of evidence $E$ that $p(E / T 1 B)$ > p(ElT2B), then E strongly favours T1 over T2.

[2] The likelihood that a life-permitting universe exists on naturalism is vanishingly small.

[3] The likelihood that a life-permitting universe exists on theism is not vanishingly small.

[4] Thus, the existence of a life-permitting universe strongly favours theism over naturalism.

This formulation has criteria A, B, C, $\mathrm{D}$, and $\mathrm{F}$. Concerning criteria $\mathrm{A}$ and $\mathrm{B}$, it 
does have a clear and logically valid formulation. The formulation is a version of modus ponens, like I suggest, although Barnes has a bit longer and more complicated version. The criterion where we are different is criterion $\mathrm{E}$, where he uses naturalism and I use chance. Most naturalistic theories will say that life is a result of chance, and thus the difference is small. But 'naturalism' can mean many things, and by specifying 'chance', it becomes unquestionable that the probability is low.

When I read Barnes' article, I was happy to see that such a great scholar thought the argument should be formulated roughly the same way that I do. Due to reorganization in Theofilos, the publishing process took a long time, with the result that Barnes' article was published before this one. This article thereby lost some of its novelty, but I think it is worth publishing anyway. Firstly, the formulation is not precisely the same, and secondly, we offer different (although compatible) reasons for choosing this formulation. This means that the two articles support each other in showing that this should be the preferred formulation. In the rest of the article, I will discuss my formulation with the differences found in the formulations suggested by Craig, Collins and Bøhn.

What are the criteria to be used when discussing how the argument should be formulated? That, of course, depends on what one wants with the argument, but if we consider it as an argument to be used in the discussion of whether God exists, it should be formulated in a way that allows theists to make the best possible case for the existence of God while at the same time being open for the best possible refutation from atheists. In this article, I am guided by the desire to pre- sent a clear argument, well suited as an argument for God's existence while also open for the most common rejection, which is the multiverse reply.

In the rest of this article, I shall defend the claim that the argument should have the characteristics A, B, C, D, E, and F. I start at the top and work my way from A to $\mathrm{F}$.

\section{A) The argument should be clear}

This characteristic goes without saying, of course, but I mention it to point out that Robin Collins' version would benefit from being clearer. Craig and Bøhn have short and clear formulations with a clear logical structure, and I argue in this article that the argument can have all the benefits that Collins' formulation has while still being short and clear.

\section{B) The argument should be logically valid}

This characteristic is also an obvious virtue, although there can be interesting inductive arguments for the existence of God as well. It is also an advantage if the logical structure of the argument is clear, which it is in the case of Craig and Bøhn, but which it is to a lesser degree in the case of Collins. The advantage of a logically valid argument is that the discussion can be concentrated on the acceptability of the premises.

C) The argument should contrast the God hypothesis with a chance hypothesis as opposed to a naturalistic single-universe hypothesis

Craig and Bøhn discuss theism opposed to chance, whereas Collins discusses theism opposed to a naturalistic single-universe hypothesis. Collins chooses this focus since it is very clear that a life-friendly universe is not to be expected given the naturalistic single universe, whereas it is 
less clear whether a life-friendly universe is to be expected given a multiverse hypothesis. By focusing on the naturalistic single-universe hypothesis, Collins then has an easier time defending the claim that a life-friendly universe is more likely given theism than given the naturalistic single-universe hypothesis.

However, in discussions of fine-tuning as an argument for the existence of God, the multiverse alternative is usually among the first responses to come up. It would be good, then, to have an argument for the existence of God which deals with the most commonly suggested alternative. The multiverse discussion must be taken up in any case, as Collins also does when he writes about the fine-tuning argument. He even uses many of the same arguments, since he argues that both the universe and the best theories of a multiverse require fine-tuning. ${ }^{9}$

But then it would be good to deal with it in the context of the discussion of the second premise, as in the other formulations of the argument here presented.

What this means in practice is that when discussing premise two, the multiverse objection should be discussed as an objection to the premise. This is how Craig usually discusses the multiverse objection as an objection to his premise two, and how Collins also argues in practice, by responding with arguments like the Bolzmann brain argument, the argument that an infinite number of universes destroys scientific reasoning, or that the multiverse itself is fine-tuned in the most plausible theories about it. ${ }^{10}$

\section{D) The argument should have life-per- mittingness as opposed to fine-tuning as datum}

Fine-tuning can be defined in different ways. Robin Collins gives the following definition: 'The laws and values of the constants of physics, and the initial conditions of any universe with the same laws as our universe, must be set in a seemingly very precise way for the universe to support life'.11 Bøhn describes finetuning for rational life as laws, constants and initial conditions having unique numerical values taken from some very wide ranges of theoretically possible such values, and that only some few values taken from these ranges are compatible with rational life. ${ }^{12}$ Craig says that:

By 'fine-tuning' one means that small deviations from the actual values of the constants and quantities in question would render the universe life-prohibiting or, alternatively, that the range of life-permitting values is exquisitely narrow in comparison with the range of assumable values. ${ }^{13}$

In every case, the point is that the set of values needed for life to exist in our universe is very small compared to the set of possible values. This is put very clearly in the definition that Luke Barnes offers of fine-tuning: 'In the set of possible physics, the subset that permit the evolution of life is very small.'14 Fine-tuning thus says something about a life-permitting universe like ours compared with numerous other (as far as we know) non-existing universes.

This is important in the argument because it shows that it is unlikely that life arose by chance. However, it is not the case that, given an intention for life to occur or given theism, we should expect fine-tuning. An intention for life or theism makes us expect life, but it does not make us expect fine-tuning for life. The universe could just as well have been coarse-tuned for life, in which case life would also have existed in alternative 
universes different from ours. Whether life would have been probable in nonexisting universes or not seems irrelevant for an intention for life or for God. Finetuning for life thus does not follow from theism, which explains why nobody thought of suggesting that before the finetuning evidence showed up.

There may actually be a reason to expect that fine-tuning for life follows from theism if we assume a specific kind of theism or intention where the universe is also fine-tuned for discoverability. As mentioned, Robin Collins has proposed that the universe is also fine-tuned for discoverability, and then the fine-tuning for life could be a feature of the universe that the creator wanted to be discovered as a clue to its origin.

In any case, since an intention for rational life or the common versions of theism to a large degree make us expect a life-permitting universe, but to a very small degree make us expect fine-tuning, the fine-tuning argument should use lifepermittingness as the datum to be explained by theism or intention instead of using fine-tuning as the datum to be explained by theism.

One could of course argue that finetuning is best explained by design or intention via an inference to the best explanation. But I argue below that the likelihood approach is the best approach, and then one hypothesis is more probable than an alternative hypothesis if the first hypothesis, to a larger degree, makes us expect the data. Since a God hypothesis makes us expect life-permittingness but not fine-tuning, life-permittingness should be used as the datum to be explained.

Robin Collins uses life-permittingness in his formulation, but Bøhn and Craig use fine-tuning. Since Bøhn focuses on what to expect from an intention for rational life versus chance, it would be better to use life-permittingness, since it is otherwise unclear whether fine-tuning is to be expected from an intention for life. Craig also uses fine-tuning as datum instead of life-permittingness, but he does not use the likelihood approach. He looks for the explanation of fine-tuning, and uses 'design' instead of 'intention for rational life'.

However, Craig also argues that 'the implication of the design hypothesis is that there exists a Cosmic Designer who fine-tuned the initial conditions of the universe for intelligent life'15. As argued above, fine-tuning does not seem to be implied by either intention or design, since fine-tuning tells us that life would not have existed in alternative non-existing universes and there is no reason for a designer of our universe to design it in a way that would have made life impossible in alternative non-existing universes (unless the designer wanted to communicate the fact of fine-tuning). It just sounds like design implies fine-tuning because the word 'fine-tuning' suggests someone doing something, but the definition of the concept does not; it merely states a numerical relation between life-permitting universes and non-life-permitting universes.

E) The argument should have a specific concept of God as hypothesis as opposed to just intention or design

This point is related to the point above, but also differs. Above, the focus was on which datum is best to choose as the object of explanation or expectation. Here the focus is on how precise the hypothesis is, which helps us deduce more precisely what follows or is to be expected from the hypothesis.

Bøhn uses intention as his hypothesis, 
but specifies that it is an intention for rational life and that knowledge and ability to produce such life is also implied in the hypothesis. But he also specifies that it does not imply God or that the intention is good. ${ }^{16}$ This is probably to widen the range of possible agents who could have produced the universe, which increases the chance that there was some intention or other, instead of arguing that it was God. Given the problem of evil, it seems easier to argue that it was an intention that produced the universe rather than a good intention.

Bøhn discusses the objection that we do not know the mind of the intender and then cannot know more exactly what was intended. He replies that this is to confuse why the universe was intended with that the universe was intended. ${ }^{17}$ But the objection has a point in that the vaguer the description of the intention, the more difficult it is to assess what it implies or makes us expect: how much life should there be, where should it be, when should it arise, how should it be, etc.

Contrary to Bøhn, Craig and Collins have much more detailed theories of God that the fine-tuning argument is meant to support. Collins, for example, argues why God would be interested in creating a multiverse, making a discoverable universe, making an independent universe, etc. 18 That they do is natural, since Bøhn, unlike the other two, is not in the business of arguing for the existence of God. But it is an advantage, in any case, to have a more specific hypothesis in order to be better able to evaluate how probable it makes the evidence. As seen, in Bøhn's case there indeed seems to be a very vague link between intention for rational life and fine-tuning of the universe for rational life.

Even theism minimally defined as a good God is better than just design or intention since it allows us to deduce a lot more than what we can from just design or intention. Common versions of theism, which emphasize that a good and loving God would want to share of God's goods and participate in relationships, imply life-permittingness, since they entail someone with a motive and power to create life. Intention or design do not imply life-permittingness, but intention for rational life and design for rational life do imply life-permittingness. None of the three imply fine-tuning. But the more detailed the theism is fleshed out the better, because it gives us more opportunity to consider the coherence of the claims made.

F) The argument should use the likelihood approach as opposed to ruling out alternatives

Collins and Bøhn use the likelihood approach while Craig rules out alternatives. I will consider Craig's approach first. Craig argues against physical necessity and chance as explanations of fine-tuning and concludes that design is the explanation. Of the three alternatives, he says that they seem to exhaust the alternatives. ${ }^{19}$ Is that right?

It seems to me that a plausible alternative to physical necessity is a physical explanation that explains the different values and shows that we are wrong today in treating all possible universes as equally probable. It would not need to be a physical necessity, but could be a contingent physical explanation. Yet it would not be the chance alternative either, because the chance alternative says that fine-tuning is due to chance, but this alternative would say that fine-tuning is merely apparent because there is not a large range of equally possible values. 
It is not at all clear to me that the alternatives rule each other out, either. If we discovered that a fine-tuned universe was necessary, would it not be surprising that such a universe was physically necessary as opposed to another kind of universe? Could not the creator have made a universe where it was physically necessary for life to arise, and this be used as an argument for the existence of God? It seems better to use metaphysical necessity or logical necessity as alternatives to God, although one could probably let those metaphysical or logical necessities be part of God as well.

I will not open the big discussion on how to define different kinds of necessity, but rather use these questions raised to show that the first premise in Craig's formulation is not so obvious. I find the likelihood approach of Collins and Bøhn better suited to avoid such criticism because they can consider the relation between hypothesis and evidence in a logically valid argument without being concerned that they have exhausted all alternatives.

Craig could argue against Bøhn and Collins that they do not exhaust the alternatives but just argue in favour of one hypothesis over another. However, arguing in favour of theism over chance (including the multiverse hypothesis) is to contrast the two hypotheses that most people find to be the two most plausible options, and it is a good strategy to discuss the best alternatives.

An objection against Collins and Bøhn could be that likelihood and conditional epistemic probability are vague concepts riddled with problems. Bøhn in fact says that he finds conditional epistemic probability to be indefinable, ${ }^{20}$ but both he and Collins refer to the fact that it is widely used in science and everyday life. Collins gives a quite-detailed account of his understanding of conditional epistemic probability, leaning on Plantinga's understanding of conditional epistemic probability and discussing some objections. The discussion of how to understand probability is too big to resolve here, but given that it is so widely used, it seems acceptable to use it also in this case.

\section{A few comments on objections to the fine-} tuning argument

There are many objections one could raise to the fine-tuning argument, but these cannot be discussed here for reasons of space. Nevertheless, I will end with some comments on the objections that can be raised.

Luke Barnes does a very good job in showing how formulating the argument this way helps to avoid many of the common objections. ${ }^{21}$ Robin Collins also covers very well a wide range of objections. ${ }^{22}$ The most common objection to the fine-tuning argument is the multiverse response, which says that if there are numerous different universes, it is likely that some (like ours) should be life-permitting. Collins covers the common objections to the multiverse response, like the Boltzmann brain objection, the objection that in the best multiverse theories, the multiverse is itself fine-tuned, and that the multiverse hypothesis (presupposing an infinite number of universes) undermines rationality.

In a future article, I hope to develop a new response to the multiverse objection. My argument will be that either there is just one universe, in which case it is most likely designed by God, or there is a multiverse generated by some multiverse generator with the capacity for actualizing infinitely many possibilities. In the latter case, I will argue that it is highly likely that one of the possibilities the multiverse 
generator has actualized is a mind connected to the generator itself. But if the multiverse generator has a mind, one could argue that it deserves to be called God. The conclusion is then that regardless of whether one assumes a universe or a multiverse, one should assume that it is created by a source with a mind.

Given that I do not think that the multiverse is a good objection to the finetuning argument, what is the best objection? I think that the best objection is that we have very little knowledge about why the parameters of the universe are as they are, which again means that our basis for establishing their probability is very thin. The future may offer a naturalistic explanation to fine-tuning, but of course, the future may also do the opposite. What matters is what conclusion is best justified today by the fine-tuning argument, and then I think theism is clearly the best-justified conclusion.

\section{Conclusion}

There is a way of combining the virtues I have argued for in this article into a new formulation of the fine-tuning argument for the existence of God. It leans quite heavily on the formulation offered by Einar Duenger Bøhn, but importantly replaces fine-tuning with life-permittingness and intention with God. The formulation is as follows:
$\mathrm{L}=$ Life-permittingness, $\mathrm{T}=$ Theism, $\mathrm{C}=$ Chance, $\operatorname{Pr}=$ Probability 1) $\operatorname{Pr}(\mathrm{L} \mid \mathrm{T})>\operatorname{Pr}(\mathrm{L} \mid \mathrm{C}) \quad \operatorname{Pr}(\mathrm{T} \mid \mathrm{L})>\operatorname{Pr}(\mathrm{C} \mid \mathrm{L})$ (Instance of the likelihood principle)

2) $\operatorname{Pr}(\mathrm{L} / \mathrm{T})>\operatorname{Pr}(\mathrm{L} / \mathrm{C})$

(Premise)

3) $\operatorname{Pr}(\mathrm{T} \mid \mathrm{L})>\operatorname{Pr}(\mathrm{Cl} \mathrm{L})$ $(1,2, \mathrm{MP})$

In full sentences, the argument says that if the probability of life-permittingness given theism is greater than the probability of life-permittingness given a chance hypothesis, then the likelihood of theism given life-permittingness is greater than the likelihood of a chance hypothesis given life-permittingness. Since in fact the probability of life-permittingness given theism is more probable than the probability of life-permittingness given a chance hypothesis, it follows that the likelihood of theism given life-permittingness is greater than the likelihood of a chance hypothesis given life-permittingness.

This way of formulating the argument is a bit tedious and clumsy, but shows explicitly that it is a deductive argument. The main idea is to use the terms theism, chance and life-permittingness, and say that since life-permittingness is more probable given theism than chance, life-permittingness favours theism over chance in this respect.

\section{Notes}

1 This is my experience from having participated in several public debates on the existence of God, and also expressed in for example Sean M. Carroll, The Big Picture: On the Origins of Life, Meaning, and the Universe Itself (New York: Dutton, 2016), 303.

2 Roger Penrose, The Road to Reality: A Complete Guide to the Laws of the Universe (London: Jonathan Cape, 2004), 762-65.

3 Robin Collins, 'The Teleological Argument: An Exploration of the Fine-Tuning of the Universe,' in The Blackwell Companion to Natural Theology, ed. William Lane Craig and James Porter Moreland (Malden, MA: Wiley-Blackwell, 2009) ; William Lane Craig, Reasonable Faith: Christian Truth and Apologetics, 3rd ed. (Wheaton, IL: Crossway Books, 2008).

4 Craig, 161.

5 Collins, 207. The formulation is similar to the approach chosen by Richard Swinburne, in Richard Swinburne, The Existence of God, 2nd ed. (Oxford: Clarendon Press, 2004), 184. 
${ }^{6}$ Einar Duenger Bøhn, 'Why This Universe?,' in Talking Seriously About God, ed. Asle Eikrem and Atle Ottesen Søvik, Nordic Studies in Theology (Zürich: LIT Verlag, 2016).

7 Collins, 205. I follow Collins in using a restricted version of the likelihood principle, which adds that the hypothesis must not be ad-hoc (ibid., 202, 06.).

${ }^{8}$ Luke A. Barnes, 'A Reasonable Little Question: A Formulation of the Fine-Tuning Argument,' Ergo: An Open Access Journal of Philosophy 6 (2019): 1225.

9 Collins, 262-69.

10 Ibid., 256-72.

11 Ibid., 204.

12 Bøhn, 130.

13 Craig, 158.

14 Luke Barnes, 'The Fine-Tuning of the Universe for Intelligent Life,' Publications of the Astronomical Society of Australia 29, no. 4 (2012): 529.

15 James Porter Moreland and William Lane Craig, Philosophical Foundations for a Christian Worldview (Downers Grove, IL: InterVarsity Press, 2003), 489.

16 Bøhn, 130.

17 Ibid., 134.

18 Robin Collins, 'Home Page of Robin Collins,' http://home.messiah.edu/ rcollins/.

19 Craig, 161.

20 Bøhn, 134.

21 Barnes, 1241-51.

22 Collins, 'The Teleological Argument: An Exploration of the Fine-Tuning of the Universe.' 